\title{
Educação sexual para adolescentes em meio a pandemia de COVID-19: Relato de
}

\section{Projeto de Extensão}

\author{
Sex education for teenagers during the COVID-19 pandemic: Report of an Extension Project \\ Educación sexual para adolescentes en medio de la pandemia de la COVID-19: Informe de un \\ Proyecto de Extensión
}

Recebido: 26/01/2022 | Revisado: 02/02/2022 | Aceito: 19/02/2022 | Publicado: 01/03/2022

Jessica Corrêa Pantoja

ORCID: https://orcid.org/0000-0001-5714-7909

Centro Universitário São Camilo, Brasil

E-mail: jessicacorreapantoja@ gmail.com

Beatriz Mynssen Marques

ORCID: https://orcid.org/0000-0001-5964-9873 Centro Universitário São Camilo, Brasil

E-mail: mynssen.bia@gmail.com

Hillary Krystie Ferreira Borges

ORCID: https://orcid.org/0000-0002-3774-1756 Centro Universitário São Camilo, Brasil

E-mail: hillaryfborges@gmail.com

Thaís Ferreira Miguel

ORCID: https://orcid.org/0000-0002-9175-3029 Centro Universitário São Camilo, Brasil

E-mail: thaisfmiguel@gmail.com

Maria Eduarda Cury Fagotti

ORCID: https://orcid.org/0000-0003-1079-5612 Centro Universitário São Camilo, Brasil

E-mail: mariaeduardafagotti@gmail.com

Danilo Munerato Barbosa

ORCID: https://orcid.org/0000-0003-4166-7090 Centro Universitário São Camilo, Brasil

E-mail: danilomb2000@gmail.com

Ana Beatriz Brito Garcia

ORCID: https://orcid.org/0000-0002-8196-4674 Centro Universitário São Camilo, Brasil

E-mail: anabiag@uol.com.br

Luciana Martins Santana Sampaio

ORCID: https://orcid.org/0000-0002-4584-4456 Centro Universitário São Camilo, Brasil

E-mail: lucianamssampaio@yahoo.com.br Maria Clara Trevisan Ribeiro

ORCID: https://orcid.org/0000-0001-6146-3082 Centro Universitário São Camilo, Brasil E-mail: ctrevisan09@gmail.com

Silvia Martinez

ORCID: https://orcid.org/0000-0003-4808-6782 Centro Universitário São Camilo, Brasil

E-mail: silvia.martinez@prof.saocamilo-sp.br

\section{Resumo}

Em decorrência da pandemia COVID-19, acentuou-se o número de gestações não planejadas entre adolescentes, Infecções Sexualmente Transmissíveis (ISTs) e abusos contra menores de idade. Evidenciou-se, então, a importância da abordagem de temáticas relacionadas à educação sexual a fim de aguçar o senso crítico dos adolescentes sobre tais conteúdos e promover, assim, uma conscientização ampla. Objetivo: Sanar as principais dúvidas acerca de vários temas primordiais para o crescimento do indivíduo em direção a sua identidade adulta, inserção na estrutura social e determinação de sua autoestima e relações afetivas, através do desenvolvimento do senso crítico de adolescentes a respeito da educação sexual. Métodos: Trata-se de um relato de experiência do Projeto "Construindo a educação sexual em um cenário durante e pós-pandemia”. Resultados: Foram atingidos 295 alunos do Ensino Fundamental e Médio, com idades entre 12 e 18 anos, durante 2021. Através do projeto, foram desenvolvidas aulas e rodas de conversa, visando tirar dúvidas e instruir os alunos. As palestras se dividiram nos seguintes temas: apresentação do projeto e questionamento para trazer as principais dúvidas dos jovens; discussão acerca do questionário e aula sobre consentimento; aula sobre ISTs e aula sobre Métodos Contraceptivos. Considerações Finais: Notou-se ao decorrer das 
aulas inúmeros tabus histórico-culturais, principalmente porque famílias seguem privando seus filhos da educação sexual emancipatória, pelo valor negativo que é atribuído a sexualidade, o que demonstra a fundamentalidade do projeto, principalmente para o desenvolvimento da sexualidade, o amadurecimento individual e coletivo, além de favorecer na construção e desenvolvimento das eventuais relações afetivas.

Palavras-chave: Adolescente; SARS-CoV-2; Educação sexual; Pandemia; Saúde reprodutiva e sexual.

\begin{abstract}
As a result of the COVID-19 pandemic, the number of unplanned pregnancies among adolescents, Sexually Transmitted Infections (STIs) and abuse against minors has increased. The importance of addressing issues related to sex education was then evidenced to sharpen adolescents' critical sense of such content and thus promote a broad awareness. Objective: To solve the main doubts about several fundamental themes for the individual's growth towards his adult identity, insertion in the social structure and determination of his self-esteem and affective relationships, through the development of the critical sense of adolescents regarding sex education. Methods: This is an experience report of the Project "Building sexual education in a scenario during the post-pandemic". Results: A total of 295 elementary and high school students, aged between 12 and 18 years old, were reached during 2021. Through the project, classes and conversation circles were developed, aiming to clarify doubts and instruct students. The lectures were divided into the following themes: project presentation and questioning to bring up the main doubts of young people; discussion about the questionnaire and class about consent; class on Sexually Transmitted Infections and class on Contraceptive Methods. Final Considerations: During the classes, numerous historical and cultural taboos were noted, mainly because families continue to deprive their children of emancipatory sexual education, due to the negative value attributed to sexuality, which demonstrates the fundamentality of the project, mainly for the development of sexuality, individual and collective maturation, in addition to favoring the construction and development of possible affective relationships.
\end{abstract}

Keywords: Adolescent; SARS-CoV-2; Sex education; Pandemic; Reproductive and sexual health.

\title{
Resumen
}

A raíz de la pandemia del COVID-19, se ha incrementado el número de embarazos no planificados en adolescentes, Infecciones de Transmisión Sexual (ITS) y maltrato a menores. Se evidenció entonces la importancia de abordar temas relacionados con la educación sexual para agudizar el sentido crítico de los adolescentes frente a dichos contenidos y así promover una amplia conciencia. Objetivo: Resolver las principales dudas sobre varios temas fundamentales para el crecimiento del individuo hacia su identidad adulta, inserción en la estructura social y determinación de su autoestima y relaciones afectivas, a través del desarrollo del sentido crítico de los adolescentes frente a la educación sexual. Métodos: Este es un relato de experiencia del Proyecto "Construyendo la educación sexual en un escenario durante y después de la pandemia". Resultados: Fueron alcanzados 295 estudiantes de enseñanza básica y media, con edades entre 12 y 18 años, durante 2021. A través del proyecto, se desarrollaron clases y círculos de conversación, con el objetivo de aclarar dudas e instruir a los estudiantes. Las conferencias se dividieron en los siguientes temas: presentación del proyecto y cuestionamiento para plantear las principales dudas de los jóvenes; discusión sobre el cuestionario y clase sobre el consentimiento; clase sobre Infecciones de Transmisión Sexual y clase sobre Métodos Anticonceptivos. Consideraciones Finales: Durante las clases se constataron numerosos tabúes históricos y culturales, principalmente porque las familias continúan privando a sus hijos de una educación sexual emancipadora, debido al valor negativo atribuido a la sexualidad, lo que demuestra la fundamentalidad del proyecto, principalmente para el desarrollo de la sexualidad, la maduración individual y colectiva, además de favorecer la construcción y desarrollo de posibles relaciones afectivas.

Palabras clave: Adolescente; SARS-CoV-2; Educación sexual; Pandemia; Salud reproductiva y sexual.

\section{Introdução}

A pandemia da COVID-19, ocasionada pelo coronavírus (SARS-CoV-2) tem se apresentado como um dos maiores desafios sanitários em escala global do século (Werneck, 2020). As dificuldades advindas dessa crise sanitária ainda inestimável para o Brasil, já está sendo sentida amplamente pelos jovens, principalmente porque são os mais afetados por uma série de impactos secundários, como: (1) demora para a vacinação, que mesmo com a antecipação sofreu inúmeros atrasos (Tabela 1); (2) dificuldades de acompanhar as atividades escolares, principalmente quando estão em situação mais vulnerável; (3) danos incalculáveis para a saúde física e mental, seja pela deficiência local de espaços para a prática de atividades físicas e cuidados envolvendo a saúde psicológica que ainda é de difícil acesso e (4) os serviços de saúde sexual e reprodutiva também foram interrompidos durante o período de isolamento, ou seja, as disrupções socioeconômicas aumentaram consideravelmente o risco de violência doméstica, o torna o lar, também um ambiente inseguro para os adolescentes (OPAS, 2021). 
Tabela 1: Cronograma vacinal dos adolescentes no estado de São Paulo.

\begin{tabular}{|c|c|c|}
\hline \multicolumn{3}{|c|}{ Cronograma de Vacinação Adolescentes do Estado de São Paulo (2021) } \\
\hline Meses & Datas & Idades Compreendidas \\
\hline \multirow{4}{*}{ Agosto } & 10 a 16 de agosto & Entre 18 e 24 anos \\
\hline & 18 a 25 de agosto & $\begin{array}{c}\text { Entre } 16 \text { e } 17 \text { anos (com deficiência, comorbidades, gestantes e } \\
\text { puérperas) }\end{array}$ \\
\hline & 30 de agosto a 5 de setembro & Entre 15 e 17 anos \\
\hline & 26 a 29 de agosto & $\begin{array}{c}\text { Entre } 12 \text { e } 15 \text { anos (com deficiência, comorbidades, gestantes e } \\
\text { puérperas) }\end{array}$ \\
\hline \multirow{2}{*}{ Setembro } & 30 de agosto a 5 de setembro & Entre 15 e 17 anos \\
\hline & 6 a 12 de setembro & Entre 12 e 14 anos \\
\hline
\end{tabular}

Fonte: Adaptação nossa do site Vacina Já do Estado de São Paulo (2022).

O aumento considerável de gestações não planejadas entre adolescentes, as Infecções Sexualmente Transmissíveis (ISTs) e abusos contra menores de idade são problemas evidenciados, que seguem causando danos sem precedentes (Febrasgo, 2021). Dessa forma, torna-se de extrema importância a abordagem de temáticas relacionadas à educação sexual a fim de aguçar o senso crítico dos adolescentes sobre tais conteúdos e promover, assim, uma conscientização ampla.

É essencial o desenvolvimento da sexualidade para o crescimento do indivíduo em direção a sua identidade adulta, inserção na estrutura social e determinação de sua autoestima e relações afetivas. Entretanto, as alterações no comportamento dos adolescentes em relação à sexualidade exigem atenção redobrada por parte dos pais e profissionais em decorrência das repercussões que incluem as vulnerabilidades relacionadas à saúde reprodutiva (Hercowitz, 2002 como citado em Alencar et al., 2008).

Com base nesses dados e conforme a Base Nacional Comum Curricular (BNCC), os discentes do Centro Universitário São Camilo (SP), realizaram o Projeto "Construindo a educação sexual em um cenário durante e pós-pandemia”, abrangendo os alunos do Ensino Fundamental e Médio, durante 2021, mesmo com todos os impasses consequentes da pandemia de COVID-19 na cidade de São Paulo (SP).

A imprescindibilidade do projeto principalmente analisando o contexto posterior ao coronavírus, visto que a pandemia segue em curso, demonstra a necessidade da manutenção do tema envolvendo a educação sexual a longo prazo, até porque a falta de planejamento familiar tem impactos negativos não exclusivamente na esfera individual, mas sobretudo na saúde pública (Fujita Jr., 2020).

Um dos principais objetivos da realização do projeto, foi sanar as dúvidas acerca de vários temas fundamentais para o amadurecimento individual, favorecendo assim na inserção na estrutura social e determinação de sua autoestima e relações afetivas, através do desenvolver o senso crítico de adolescentes a respeito da educação sexual, com o apoio da coordenação e professores da escola que foram fundamentais para execução do mesmo.

\section{Metodologia}

Trata-se de um estudo descritivo-exploratório, tipo de relato de experiência, no qual foram realizadas idas à comunidade parceira por discentes do Curso de Medicina (Tabela 2) e foram realizadas discussões através de rodas de diálogo, palestras e demonstração ativa (Figura 1) de métodos contraceptivos. 
Tabela 2: Participantes do projeto do Centro Universitário São Camilo.

\begin{tabular}{|c|c|}
\hline Nível de Ensino/Curso & Quantidade \\
\hline Discentes de Medicina & 9 \\
\hline Docente/Orientadora & 1 \\
\hline Total & 10 \\
\hline
\end{tabular}

Fonte: Autores (2022).

O projeto foi aprovado em conformidade com o Edital Práticas de Extensão no 043/2020 do Centro Universitário São Camilo (2020), no qual o principal objetivo que foi atingido durante o mapeamento local da comunidade, a proposta da elaboração das aulas com base nos dados levantados e a organização e tabulação de dados.

Figura 1: Kit com exemplares de métodos contraceptivos (pílulas anticoncepcionais estrogênio, pílulas anticoncepcionais progesterona, pílulas anticoncepcionais combinados, DIU de cobre, DIU de cobre + prata, DIU KYLEENA, DIU MIRENA, Anel vaginal, implante subdérmico, preservativos femininos, preservativos masculinos, adesivos anticoncepcionais), disponibilizado pela universidade.

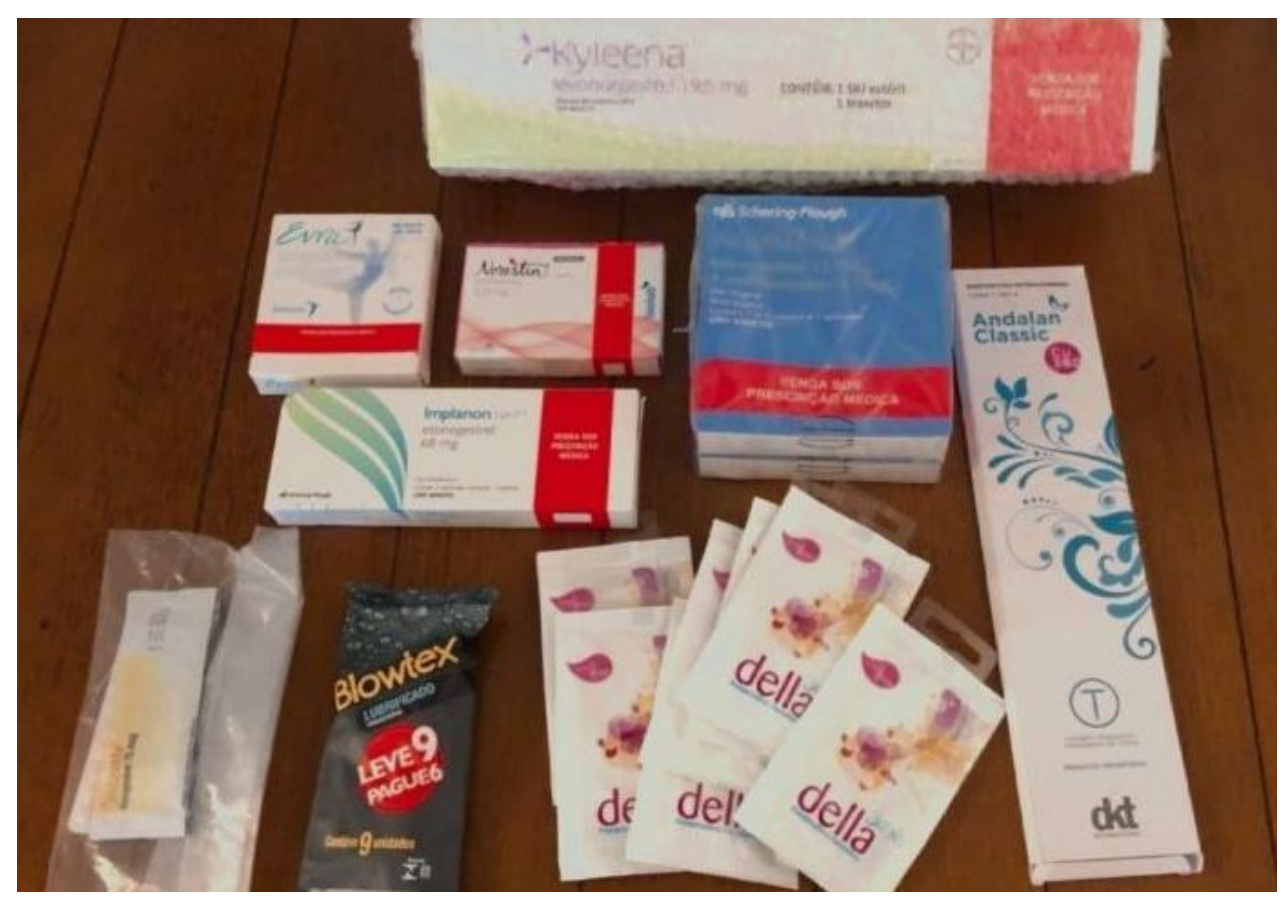

Fonte: Autores (2022).

A escola escolhida é estadual, integral no estado de São Paulo, em uma região central e urbana, e o foco são unicamente alunos do ensino fundamental e médio (Tabela 4). O diálogo com a coordenação pedagógica ocorreu principalmente porque a mesma já desenvolve projetos sociais com outras instituições de ensino superior de outros cursos. A faixa etária do público-alvo foram de adolescentes (12 aos 18 anos, segundo o Estatuto da Criança e do Adolescente (ECA), a Lei 8.069 de 1990), escolhidos sobretudo pelos índices de gravidez precoce em São Paulo, que afeta a escolaridade e empregabilidade da mulher, além aumenta os índices de prematuridade das crianças nascidas vivas, e como consequência o 
trabalho infantil (Mapa da Desigualdade da Primeira Infância, 2020).

O cronograma (Tabela 3) foi realizado em conformidade com o retorno das escolas municipais e as equipes dos discentes foram ministrar as aulas nos turnos matutino e vespertino, com apoio dos professores de ciências (ensino fundamental), biologia e química (ensino médio), por recomendação da coordenação escolar.

Tabela 3: Desenvolvimento do projeto durante os anos de 2021 e 2022.

\begin{tabular}{|c|c|c|c|c|c|c|}
\hline \multicolumn{7}{|c|}{ Cronograma das atividades desenvolvidas } \\
\hline Meses e Ano/Elaboração & Jan.-Ago./2021 & Set. /2021 & Out./2021 & Nov./2021 & Dez./2021 & Jan./2022 \\
\hline $\begin{array}{c}\text { Período de preparação e } \\
\text { elaboração }\end{array}$ & $\mathrm{X}$ & $\mathrm{X}$ & $\mathrm{X}$ & $\mathrm{X}$ & $\mathrm{X}$ & \\
\hline $\begin{array}{c}\text { Apresentação do material } \\
\text { para a comunidade }\end{array}$ & & $\mathrm{X}$ & $\mathrm{X}$ & $\mathrm{X}$ & $\mathrm{X}$ & \\
\hline Execução do projeto & & & & $\mathrm{X}$ & $\mathrm{X}$ \\
\hline Apresentação dos resultados & & & & & \\
\hline
\end{tabular}

Fonte: Autores (2022).

Uma das carências descritas pela coordenadora pedagógica são "o apoio e a orientação sobre cuidados com a saúde e sexualidade em parceria com a escola", e um dos problemas levantados foi a necessidade do diálogo envolvendo consentimento e relacionamento, além de casos eventuais que ocorrem de gravidez durante o ensino médio e acabam por atrapalhar/interromper os estudos.

Tabela 4: Quantidade aproximada de alunos por ano/série, que foram abrangidos pelo projeto. Número disponibilizado pela coordenação pedagógica.

\begin{tabular}{|c|c|}
\hline Ano/Série & $\begin{array}{c}\text { Quantidade de } \\
\text { alunos }\end{array}$ \\
\hline $6^{\text {o }}$ Ano Ensino Fundamental & 39 \\
\hline $7^{\mathbf{0}}$ Ano Ensino Fundamental & 38 \\
\hline $8^{\text {o }}$ Ano Ensino Fundamental & 38 \\
\hline $9^{\circ}$ Ano Ensino Fundamental & 38 \\
\hline $1^{\text {a }}$ Série do Ensino Médio & 42 \\
\hline $2^{\text {a }}$ Série do Ensino Médio/Primeira Turma & 38 \\
\hline $2^{\text {a }}$ Série do Ensino Médio/Segunda Turma & 40 \\
\hline $3^{\text {a }}$ Série do Ensino Médio & 41 \\
\hline Total de alunos & 295 \\
\hline
\end{tabular}

Fonte: Autores (2022). 


\section{Resultados e Discussão}

Em decorrência da pandemia, durante o ano de 2020 o ensino foi completamente remoto na escola escolhida. A priori, o projeto foi desenvolvido de forma híbrida, semelhante as aulas da comunidade, contudo, graças a retomada das aulas e atendendo as regras sanitárias gerais e obrigatórias, visando atingir ao máximo o público (Tabela 4), que nem sempre obteve acesso à internet, computador em sua própria residência, e muitas tendo que se locomover a escola para utilizar durante o período em questão, as conversas foram presenciais.

\subsection{Realização do Projeto Presencial em meio a Pandemia de COVID-19 no Estado de São Paulo}

Segundo Munhoz (2020) as aulas nas redes estadual e municipal de ensino público de São Paulo começaram as serem suspensas gradativamente em março de 2020, em virtude da confirmação do primeiro caso de transmissão comunitária do vírus na cidade. Desde então o processo de adaptação do ensino remoto e retorno as atividades convencionais nas escolas, tem sido amplamente discutido, principalmente por conta do isolamento social e dos protocolos sanitários (Tabela 5).

Tabela 5: Relação das aulas ministradas no colégio parceiro.

\begin{tabular}{|c|c|c|c|c|c|}
\hline \multicolumn{7}{|c|}{ Realização das Aulas durante o ano de 2021 } \\
\hline $\begin{array}{c}\text { Apresentação do projeto disponibilização do } \\
\text { questionário }\end{array}$ & $13 / 09$ & $27 / 09$ & $08 / 11$ & $22 / 11$ & $29 / 11$ \\
\hline $\begin{array}{c}\text { Discussão acerca do questionário e Aula } \\
\text { sobre consentimento }\end{array}$ & & X & & & \\
\hline $\begin{array}{c}\text { Aula sobre Infecções Sexualmente } \\
\text { Transmissíveis (IST) }\end{array}$ & & & & & \\
\hline Aula sobre Métodos Contraceptivos & & & & & \\
\hline
\end{tabular}

Fonte: Autores (2022).

A execução das aulas foi desenvolvida em todos os cenários possíveis levando em consideração sobretudo a quantidade contágios (Figura 2) e óbitos (Figura 3), o que foi fundamental para o cuidado dos alunos e dos professores da escola, já que algumas aulas da universidade continuaram sendo práticas. É importante frisar que o vírus possui variantes (Instituto Butantã, 2021), e as incertezas envolvendo o SARS-CoV-2 e o trabalho com a comunidade necessitaram de cuidados redobrados (Borges, 2020).

A busca por dados exatos foi fundamental para execução, afinal segundo (Jones, 2020):

Embora seja difícil mensurar qualquer pandemia enquanto ela ainda está em curso, ainda mais quando se trata de uma doença nova, a tarefa de conhecer a real extensão de sua gravidade torna-se mais complicada em razão de certas especificidades do novo coronavírus, como a grande proporção de indivíduos infectados, mas assintomáticos, as variadas manifestações clínicas da doença e as peculiaridades da resposta imune ao Sars-CoV-2. 
Figura 2: Quantidade de casos por dia de COVID-19 no estado de São Paulo no período de 2020 a 2022 o que demonstra uma queda considerável durante a realização do projeto nos meses de setembro e novembro de 2021.

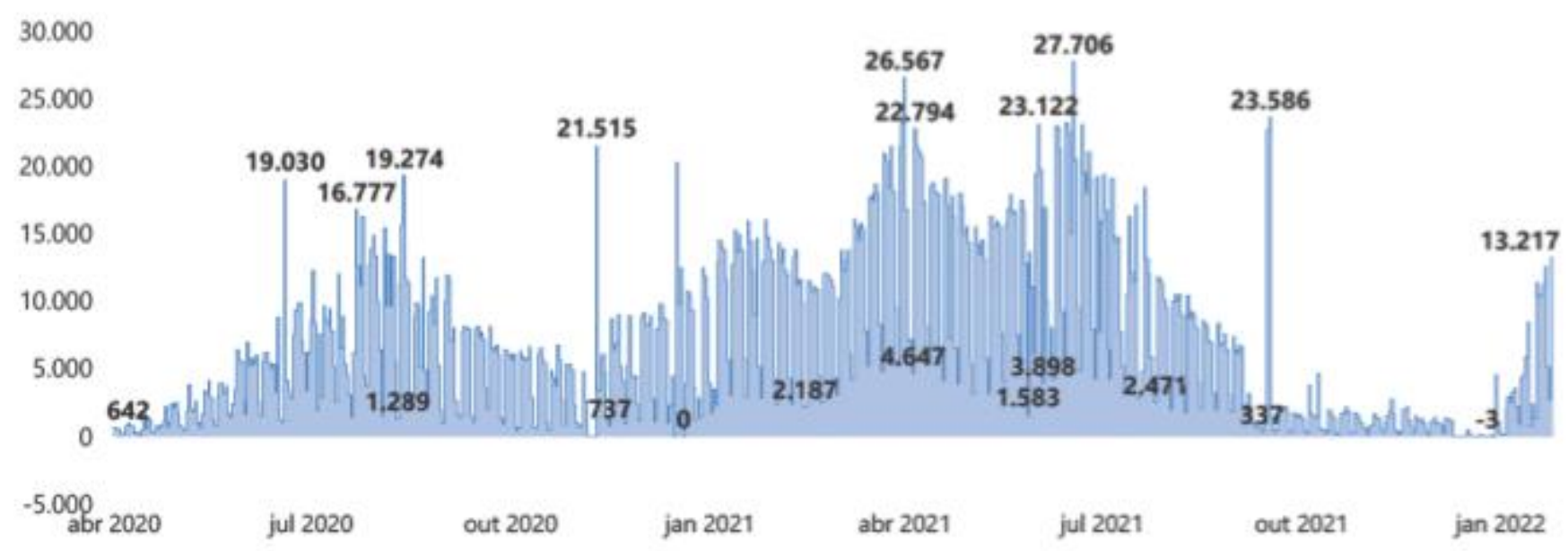

Fonte: Fundação Sistema Estadual de Análise de Dados (SEADE) (2022).

Figura 3: Índice de óbitos do vírus no estado de São Paulo no período de 2020 a 2022, demonstrando que a realização do projeto presencial entre os meses de setembro e novembro de 2021 as taxas estavam em baixa.

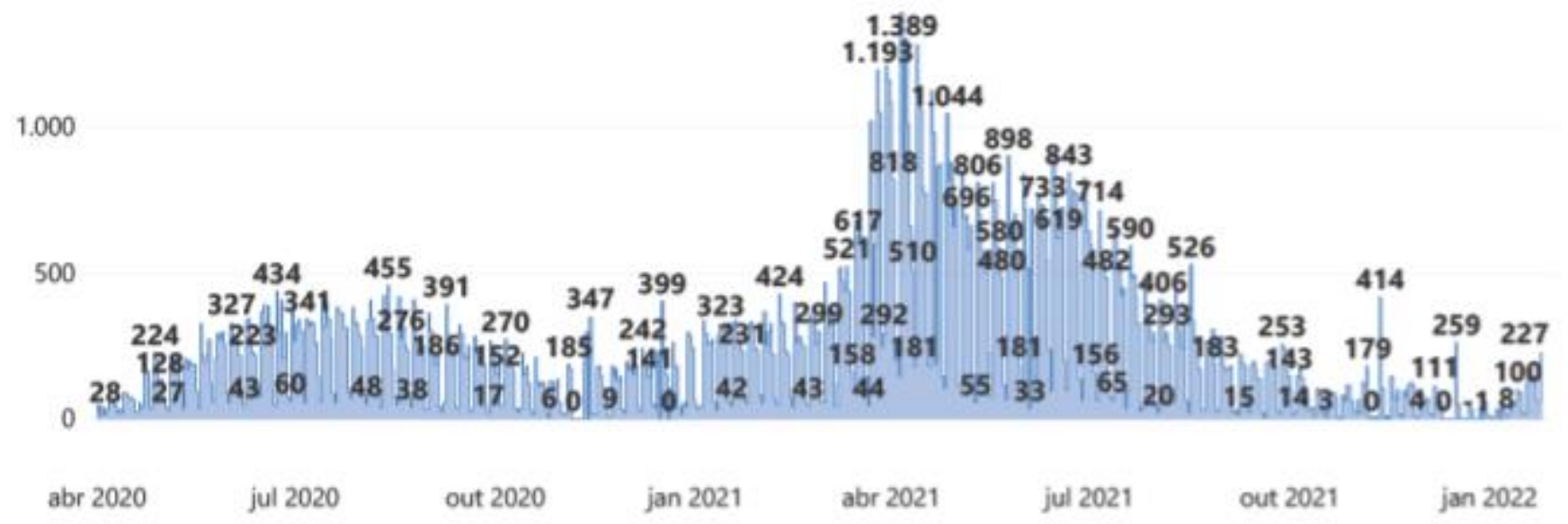

Fonte: Fundação Sistema Estadual de Análise de Dados (SEADE) (2022).

\subsection{Atividades Desenvolvidas para Comunidade}

Visando o melhor aproveitamento dos jovens, dividiu-se o conteúdo em quatro aulas assuntos medulares: (1) apresentação dos participantes do projeto e questionário para desenvolver o conteúdo e incentivar a erudição; (2) resolução do questionário e aula sobre consentimento; (3) aula sobre ISTs e (4) encerramento com aula sobre Métodos Contraceptivos e apresentação de materiais disponibilizados pelo Centro Universitário São Camilo (Imagem 1).

Durante o primeiro encontro, além de conhecer o público-alvo, foi analisado entendimento prévio dos adolescentes, com a aplicação de questionário com principais tópicos acerca do tema proposto do projeto, para, assim, delimitar uma melhor abordagem. No segundo, o questionário foi respondido adequadamente e iniciou-se então as aula sobre consentimento com materiais das educadoras Caroline Arcari Meyer e Maria Julieta Correia Jacob (Editora Caqui, 2022), que possuem um amplo acervo, de várias obras relacionadas ao assunto em questão disponível, que se encaixa nas normas pedagógicas atuais do 
Ministério da Educação (2018).

O terceiro encontro buscou conscientização acerca das principais ISTs, que apresentam altas taxas epidemiológicas de incidência e prevalência entre os jovens (Nery et al., 2015). Foram discutidas as formas de transmissão, sinais e sintomas, diagnóstico, métodos de prevenção e cura das infecções: sífilis, HPV, HIV/AIDS. Além disso, a diferença entre a denominação ISTs e Doenças Sexualmente Transmissíveis (DST), uma vez que se faz necessário entender que são conceitos diferentes e DST não é mais utilizado (Departamento de IST, Aids e Hepatites Virais, 2016).

Durante o último encontro, além da aula sobre Métodos Contraceptivos, apresentou-se materiais disponibilizados pela universidade para estímulo visual e prático, não apenas teórico, sendo possível finalizar o projeto com uma participação efetiva da comunidade, que convidou a equipe para realizações de parcerias futuras.

\section{Considerações Finais}

Furlanetto et al. (2018) salienta do espaço escolar, tendo em vista que o mesmo cumpre uma função social responsável pela evolução intelectual, física, social e cultural dos indivíduos. A sexualidade, por sua vez, transcorre todos as fases do desenvolvimento dos alunos e depende diretamente das características da formação discente e dos diferentes modelos aprendidos na família e na escola através de professores e funcionários. Assim, ao disponibilizarmos informações e um espaço no qual ocorreu uma comunicação fluida e troca de conhecimento, acreditamos que foi possível atingir os alunos, diretamente ou indiretamente e que os mesmos terão influência sobre a própria comunidade.

Para Sfair et al. (2015), a educação sexual deve, antes de tudo, fortalecer adolescentes, fomentar o sentimento de estima relacionado ao seu corpo, aos valores pelos quais opta, sensibilizando cada um para que sinta o mesmo em relação ao outro, mesmo quando este lhe seja díspar. O tema central foi desmistificado, sobretudo através de um esclarecimento, a partir da proposta inicial, e seguindo uma abordagem que antecipa não apenas os riscos do exercício da sexualidade, mas também os sentimentos, o respeito e a responsabilidade envolvidos.

Desse modo, é notório que o projeto alcançou os objetivos propostos - ainda que precise de um maior aprofundamento seja da comunidade visitada ou acadêmica que proporcionou o curso - processo esse basilar para o desenvolvimento individual, visto que as aulas realizadas foram sintetizadas, seja por conta da quantidade de alunos da escola (295 adolescentes) ou pela quantidade de alunos participantes das atividades desenvolvidas (9 discentes de medicina) e tempo corrido em meio a pandemia que impossibilitaram um maior contato e diálogo direto com os estudantes.

Eventuais distrações e momentos de dispersão foram comuns, principalmente quanto a assuntos relacionadas a dúvidas fundamentais, estas misturadas com vergonha e timidez por parte dos alunos, o que demonstrava interesse constante; questionamentos, inclusive após as discussões sobre o uso de preservativos e as idas as ginecologistas e envolvendo o consentimento.

As limitações que atrasaram o desenvolvimento do projeto presencialmente trouxeram inúmeros questionamentos principalmente voltados a acessibilidade e disponibilidade do que foi produzido para a população, tendo em vista a sua indispensabilidade a longo prazo. Afinal, a escola tida como uma instituição basilar para o indivíduo e sua constituição, tem como uma das tarefas mais imprescindíveis, seja para pais, alunos e professores, também ensinar os jovens a viverem e superarem as adversidades em um mundo que segue passando por mudanças constantes, com diversas incompatibilidades interpessoais, cooperando para o processo de desenvolvimento dos indivíduos (Dessen \& Polonia, 2007).

Evidenciou-se enfim uma vontade inerente de mudança em cada um deles, o que demonstrou que o futuro é argumentador e revolucionário, afinal o estímulo à participação dos estudantes na escola é essencial para a assimilação da cidadania, e para sentir-se sujeito do processo educacional, e a escola cidadã deve partir da necessidade dos alunos, na defesa de uma educação dialógica (Freire, 2018). E para que o processo de formação seja completo, a formação crítica necessita ser 
vivenciada e a execução do projeto de extensão proporcionou, felizmente, essa troca mútua com êxito.

\section{Referências}

Arruda, S., Ricardo, C., Nascimento, M., \& Fonseca, V. (2010). Guia adolescentes, jovens e educação em sexualidade. Promundo-US. https://promundoglobal.org/resources/guia-adolescentes-jovens-e-educacao-em-sexualidade/

Borges, L. (2020). Vacinados podem transmitir a doença, ainda que estejam imunes à COVID? VEJA. https://veja.abril.com.br/coluna/diario-davacina/vacinados-podem-transmitir-a-doenca-ainda-que-estejam-imunes-a-covid/

Brasil. Governo de São Paulo. (2022) Vacina Já. Cronograma de vacinação. https://www.vacinaja.sp.gov.br/

Brasil. Departamento de Atenção Básica. (2010). Saúde sexual e saúde reprodutiva. Ministério da Saúde.

Brasil. Ministério da Saúde. (2018). Base Curricular Nacional http://basenacionalcomum.mec.gov.br/images/BNCC_EI_EF_110518_versaofinal_site.pdf

Brasil. Ministério da Saúde. (2008). Guia para a formação de profissionais de saúde e de educação: saúde e prevenção nas escolas. Ministério da Saúde, Secretaria de Vigilância Em Saúde, Programa Nacional de DST e AIDS.

Brasil. Ministério da Saúde. Secretaria de Atenção à Saúde. Departamento de Ações Programáticas Estratégicas. (2006). Direitos sexuais, direitos reprodutivos e métodos anticoncepcionais. Ministério Da Saúde.

Braz E Barros, M., \& Miranda, J. (2019). Revista Educação Pública - Abordagem do tema sexualidade no ambiente escolar. Revista Educação Pública. https://educacaopublica.cecierj.edu.br/artigos/19/4/abordagem-do-tema-sexualidade-no-ambiente-escolar

Carvalho, P. (2020). Saúde do adolescente piorou na pandemia e 76\% tiveram afastamento social. VivaBem. Retrieved December 1, 2020. https://www.uol.com.br/vivabem/noticias/redacao/2020/09/27/sbu-saude-do-adolescente-piorou-na-pandemia-e-76-teve-afastamento-social.htm

Cavasin, Sylvia, et al. Orientações Técnicas de Educação Em Sexualidade Para O Cenário Brasileiro: Tópicos E Objetivos de Aprendizagem. Brasília, DF, Brasil, Unesco, Organização das Nações Unidas para a Educação, a Ciência e a Cultura, Representação no Brasil, 2014.

Centro Universitário São Camilo. (2020). Edital Práticas de Extensão - 043/2020. Centro Universitário São Camilo - SP. https://saocamilosp.br/eventos/edital_extensao_praticas

Couzemenco, F. (2020). Abordagem da Covid-19 como sindemia e não pandemia salienta aspectos sociais. Século Diário. https://www.seculodiario.com.br/saude/abordagem-da-covid-19-como-sindemia-e-nao-pandemia-salienta-aspectos-sociais

Crianças e adolescentes estão sendo profundamente impactados pela pandemia de COVID-19, afirma diretora da OPAS. (2021). OPAS/OMS | Organização Pan-Americana da Saúde. https:/www.paho.org/pt/noticias/15-9-2021-criancas-e-adolescentes-estao-sendo-profundamente-impactados-pela-pandemia-covid

Departamento de IST, Aids e Hepatites Virais (2016). Departamento passa a utilizar nomenclatura “IST" no lugar de “DST. ” Departamento de Doenças de Condições Crônicas e Infecções Sexualmente Transmissíveis. http://www.aids.gov.br/pt-br/noticias/departamento-passa-utilizar-nomenclatura-ist-no-lugar-dedst

Dessen, M. A., \& Polonia, A. C. (2007). A família e a escola como contextos de desenvolvimento humano. Paidéia, 17 (36), 21-32. https://doi.org/10.1590/S0103-863X2007000100003

Editora Caqui. (2022). Quem Somos. https://www.editoracaqui.com.br/

Drauzio Varella: falta de acesso ao planejamento familiar é uma violência. (2007). Agência Senado. https://www12.senado.leg.br/noticias/materias/2007/04/10/drauzio-varella-falta-de-acesso-ao-planejamento-familiar-e-uma-violencia

FEBRASGO. Manual de Anticoncepção Manual de Anticoncepção. (2015). São Paulo: Federação Brasileira das Associações de Ginecologia e Obstetrícia. https://central3.to.gov.br/arquivo/494569/

Fernandes, F. (2019). O papel da escola no combate ao abuso sexual de crianças e adolescentes. MultiRio. http://multirio.rio.rj.gov.br/index.php/leia/reportagens-artigos/reportagens/14963-o-papel-da-escola-no-combate-ao-abuso-sexual-de-crian\%C3\%A7as-eadolescentes

Freire, P. (2018). Educação Como Prática da Liberdade. São Paulo Paz e Terra.

Fórum Brasileiro de Segurança Pública. (2020). Violência Doméstica Durante Pandemia de Covid-19 Edição 02. https://forumseguranca.org.br/publicacoes_posts/violencia-domestica-durante-pandemia-de-covid-19-edicao-02/

Fujita Jr, L. (2020). Falta de planejamento familiar. Drauzio Varella. https://drauziovarella.uol.com.br/videos/series-e-documentarios/o-sistema-07-falta-deplanejamento-familiar/

Gonçalves, R., Faleiro, J., \& Malafaia, G. (2013). Educação sexual no contexto familiar e escolar: Impasses e desafios. HOLOS, 5, 251-263. https://doi.org/10.15628/holos.2013.784

Horton, R. (2020). Offline: COVID-19 is not a pandemic. ScienceDirect. https://linkinghub.elsevier.com/retrieve/pii/S0140673620320006

Jacob, J (2018). Tuca e Juba - Prevenção de Violência Sexual Para Adolescentes. Ilustrações da Ilustralu. Recife.

Jones, F. (2020). O enigma da letalidade. Revista Pesquisa FAPESP. https://revistapesquisa.fapesp.br/o-enigma-da-letalidade/ 
Martins, E. (2020). Pandemia afasta grávidas do pré-natal e aumenta alterações comportamentais em crianças, diz pesquisa. O Globo. https://oglobo.globo.com/saude/coronavirus/pandemia-afasta-gravidas-do-pre-natal-aumenta-alteracoes-comportamentais-em-criancas-diz-pesquisa-24594358

Munhoz, F. (2020). Aulas serão suspensas em escolas estaduais e municipais de SP por coronavírus. Folha de S. Paulo. https://agora.folha.uol.com.br/saopaulo/2020/03/escolas-de-sao-paulo-terao-aulas-suspensas-por-conta-do-coronavirus.shtml

Nery, J. A. C., Sousa, M. D. G., Oliveira, E. F., \& Quaresma, M. V. (2015). Infecções sexualmente transmissíveis na adolescência. Residência Pediátrica 5(3 Supl.1), 64-78. http://residenciapediatrica.com.br/detalhes/170/infeccoes-sexualmente-transmissiveis-na

Oliveira, J. (2020). Abortos legais em hospitais referência no Brasil disparam na pandemia e expõem drama da violência sexual. El País Brasil. https://brasil.elpais.com/brasil/2020-08-30/abortos-legais-em-hospitais-referencia-no-brasil-disparam-na-pandemia-e-expoem-drama-da-violencia-sexual.html

Pesquisa mostra que a pandemia afastou homens dos consultórios médicos. (2020). O Dia. https://odia.ig.com.br/vida-saudavel/saude/2020/11/6023667pesquisa-mostra-que-a-pandemia-afastou-homens-dos-consultorios-medicos.html

Por que acontecem mutações do SARS-CoV-2 e quais as diferenças entre cada uma das variantes. (2021). Instituto Butantan. https://butantan.gov.br/noticias/por-que-acontecem-mutacoes-do-sars-cov-2-e-quais-as-diferencas-entre-cada-uma-das-variantes

Rede Nossa São Paulo \& Bernard van Leer Foundation. (2020). Mapa da Desigualdade da Primeira Infância. Rede Nossa São Paulo. https://www.nossasaopaulo.org.br/wp-content/uploads/2020/02/Mapa_PrimeiraInfancia-2020-completo.pdf

Reflexões sobre a Semana Nacional de Prevenção da Gravidez na Adolescência. (2021). Febrasgo. https://www.febrasgo.org.br/pt/noticias/item/1210reflexoes-sobre-a-semana-nacional-de-prevencao-da-gravidez-na-adolescencia-2021

Santos, M. T. (2019). 20\% das brasileiras não vão ao ginecologista com frequência. Por quê? Veja Saúde. https://saude.abril.com.br/medicina/20-dasbrasileiras-nao-vao-ao-ginecologista-com-frequencia-por-que/

Sfair, S. C., Bittar, M., \& Lopes, R. E. (2015). Educação sexual para adolescentes e jovens: mapeando proposições oficiais. Saúde e Sociedade (Impresso), 24(2): 620-632. https://pesquisa.bvsalud.org/portal/resource/pt/lil-749055

Ushikusa, T. (2020). Precisamos lidar com a gravidez não planejada em tempos de coronavírus. Veja Saúde. https://saude.abril.com.br/coluna/com-apalavra/precisamos-lidar-com-a-gravidez-nao-planejada-em-tempos-de-coronavirus/

Werneck, G. L. (2020). SciELO - Saúde Pública - A pandemia de COVID-19 no Brasil: crônica de uma crise sanitária anunciada A pandemia de COVID-19 no Brasil: crônica de uma crise sanitária anunciada. Cadernos de Saúde Pública. https://www.scielosp.org/article/csp/2020.v36n5/e00068820/\# 